\title{
Hierarchical attention in discriminating objects at different levels of specificity
}

\author{
MARY-ELLEN LARGE and PATRICIA A. MCMULLEN \\ Dalhousie University, Halifax, Nova Scotia, Canada
}

\begin{abstract}
An implicit assumption of studies in the attentional literature has been that global and local levels of attention are involved in object recognition. To investigate this assumption, a divided attention task was used in which hierarchical figures were presented to prime the subsequent discrimination of target objects at different levels of category identity (basic and subordinate). Target objects were identified among distractor objects that varied in their degree of visual similarity to the targets. Hierarchical figures were also presented at different sizes and as individual global and local elements in order to investigate whether attention-priming effects on object discrimination were due to grouping/parsing operations or spatial extent. The results showed that local processing primed subordinate object discriminations when the objects were visually similar. Global processing primed basic object discriminations, regardless of the similarity of the distractors, and subordinate object discriminations when the objects were visually dissimilar. It was proposed that global and local processing aids the selection of perceptual attributes of objects that are diagnostic for recognition and that selection is based on two mechanisms: spatial extent and grouping/parsing operations.
\end{abstract}

Since Navon's (1977) seminal article, there have been numerous research papers on the topic of global and local attention. In the bulk of this research, artificial stimuli consisting of large digits or letters made up of smaller digits or letters (hierarchical figures) have been used. An underlying assumption is that effects of global and local attention observed with hierarchical figures reflect global and local attentional allocation during object and scene recognition in the real world. It is true that objects and scenes can be conceptualized as having levels of structure that are ordered hierarchically, where global levels encompass local structural components. A forest scene can be broken down into separate trees, bushes, and other forest flora and fauna. However, the hierarchical figures traditionally employed for research into global and local attention have been artificial stimuli that have no place outside the laboratory (Navon, 2003). How can we know that observations based on artificially constructed stimuli reflect how our visual and attentional systems operate with more realistic objects and scenes? The aim of this study was to investigate whether and how global and local levels of attention influence the discrimination of objects.

A number of models have been proposed to account for the effects of global and local processing. These can be divided into two broad categories. One set of models con-

This work was supported by Natural Sciences and Engineering Research Council of Canada and Human Frontiers Science Program awards to the second author. We acknowledge David Shore for his development of the hierarchical stimuli. Correspondence concerning this article should be addressed to M.-E. Large, Department of Psychology, University of Western Ontario, Social Science Centre, London, ON, N6A 5C2 Canada (e-mail: mlarge2@uwo.ca). siders the spatial extent of attention, in which attention is focused on a region of space that can vary in size (Lamb \& Robertson, 1988; Lamb \& Yund, 2000; Robertson, 1996; Robertson, Egly, Lamb, \& Kerth, 1993). The size of the attended region would be relatively larger for global processing than for local processing. Other models are based on principles of perceptual organization where visual information is divided into global and local processing streams by grouping local elements for global identifications or by parsing local elements for local identifications (Han \& Humphreys, 2002; Han, Humphreys, \& Chen, 1999a, 1999b; Kimchi, 1998, 2000). In both models, a distinction can be made between attending to the coarse structure of an object's visual properties at the global level and attending to fine details at the local level.

This notion of coarse and fine-detailed visual information is also relevant to object categorization (Archambault, Gosselin, \& Schyns, 2000; Morrison \& Schyns, 2001; Oliva \& Schyns, 1997; Schyns \& Oliva, 1999). Objects can be categorized at various levels of specificity. At the most general level (superordinate), the object dog could be called an animal; at an intermediate level (basic), it could be called a dog; and at a specific level (subordinate), it could be called a beagle. It is at the basic level that objects are most likely to be identified and named most quickly (Jolicœur, Gluck, \& Kosslyn, 1984; Murphy \& Brownell, 1985; Murphy \& Lassaline, 1997; Rosch, Mervis, Gray, Johnson, \& Boyes-Braem, 1976). One of the reasons that subordinate categorizations may take longer is that the perceptual information required to categorize objects at this level is less discriminable than the information required for basic-level categorizations (Biederman, Subramaniam, Bar, Kalocsai, \& Fiser, 1999; Biederman, 
Subramaniam, Kalocsai, \& Bar, 1999; Jolicœur et al., 1984). In general, subordinate categorizations likely require processing of the fine visual details of an object's structure, whereas coarse-scale information may be sufficient for basic-level categorizations. Global and local processes could allocate attentional resources to facilitate the selection of fine- or coarse-scale perceptual cues diagnostic for the recognition or categorization of an object.

However, it is also important to consider the effects of visual similarity in visual tasks (Duncan \& Humphreys, 1989). It has been shown that the degree of visual similarity or dissimilarity between objects belonging to the same category can affect performance on categorization tasks. For example, responses to atypical category members are quicker at the subordinate level than at the basic level (Jolicœur et al., 1984; Murphy \& Brownell, 1985; Murphy \& Lassaline, 1997). Of course, atypical exemplars (e.g., ostrich, penguin) are generally more visually distinct from other within-category exemplars. Lloyd-Jones and Humphreys (1997a, 1997b) demonstrated that structurally similar objects (fruits and vegetables) were more difficult to name at the basic level and more difficult to categorize at the superordinate level than were structurally dissimilar objects (clothing and furniture). Their results suggested that visual similarity is not just a factor in subordinate category judgments, but also a factor in basic-level and superordinate-level category judgments. Accordingly, discriminations between visually dissimilar objects could rely on coarse-scale perceptual information, and discriminations between visually similar objects could rely on fine-scale perceptual information, independently from the category level of the object. Consequently, global and local processes in object recognition may be more responsive to degrees of visual similarity between objects than to category membership.

This research involved two experiments. The purpose of the experiments was to provide empirical evidence demonstrating that global and local processing is used in the recognition of realistic objects. More specifically, the aim was to determine whether global processing influenced the selection of coarse-grained information from the input image and local processing influenced the selection of fine-grained information. Given that the perceptual demands on the visual system may vary, depending on the nature of a recognition task (Morrison \& Schyns, 2001; Schyns, 1998), the investigation focused on whether global and local processing influenced the categorization of objects at basic and subordinate levels or whether global and local processing influenced the categorization of objects that were visually similar or dissimilar.

Second, we wanted to determine whether the use of global and local processes in discriminating realistic objects was related to the distribution of attention across a region of space (attentional window hypothesis) or to parsing/grouping processes (parsing hypothesis) occurring during recognition. Investigations examining global and local processing of hierarchical figures support the involvement of both of these mechanisms (Kim, Ivry, \& Robertson, 1999; Lamb \& Robertson, 1988; Lamb \&
Yund, 2000; Robertson, 1996; Robertson et al., 1993). Theoretically, either or both of these mechanisms could be involved in the processing of global and local features of a pictorial image. Researchers investigating the automatic activation of semantic information associated with the global form of objects have speculated that attentional resources are distributed across a region in space (Boucart \& Humphreys, 1992; Murray \& Jones, 2002). Edelman and Intrator (2003) also have postulated that access to different levels of object representations is mediated by the spatial extent of attention. Alternatively, global and local processing may play a role in the parsing of images into a hierarchy of modular parts (Biederman, 1987; Duncan \& Humphreys, 1989; Marr, 1982). Global processing could capture visual information relevant for generating input representations that match to large-scale components of an object's structure. Similarly, local processing could capture visual information relevant for generating input representations that match to small-scale components of an object's structure (Han et al., 1999a).

In order to investigate the role of global and local processing in object recognition, this research made use of a well-known effect in the attention literature: the level repetition effect. Using hierarchical stimuli to manipulate global and local levels of processing, the level repetition procedure involves comparing the performance on a second stimulus presentation when the attention levels for the first and the second stimulus presentations are the same versus when they are different. It has been found that the efficiency of detecting targets increased when the level of attention was repeated across consecutive stimulus presentations. The level repetition effect has been shown to be longlasting (Robertson, 1996; Ward, 1982) and robust to changes in target form and target location (Filoteo, Friedrich, \& Stricker, 2001; Hübner, 2000; Lamb \& Yund, 1996; Ward, 1982). In the present experiments, a priming paradigm was chosen, similar to that used in studies of level repetition effects. A global and local divided attention task preceded and primed a subsequent target discrimination task in which participants indicated the presence of a target or a distractor object at either a basic level or a subordinate level of categorization. The degree of visual similarity between the targets and the distractors in the object discrimination task was manipulated.

\section{EXPERIMENT 1}

The main purpose of Experiment 1 was to establish whether the allocation of attentional resources at global and local levels would occur in an object discrimination task and, if so, the circumstances under which they would be deployed. We have argued that coarse-scale and finescale perceptual cues are diagnostic for different recognition tasks. Granting that the allocation of attentional resources assists the processing of perceptual cues present in a stimulus input, it is possible that variations in the distribution of attentional resources will support the processing of different kinds of perceptual cues. For example, if attention is allocated to the global features of a stimulus, 
this should benefit the selection of coarse-scale perceptual cues, and if attention is allocated to local features, this should benefit the selection of fine-scale perceptual cues.

It was expected that if the priming effects of global and local attention were sensitive to the category level of target objects, global decisions should prime basic decisions, and local decisions should prime subordinate decisions, independently of the degree of visual similarity between the target objects and the distractor objects. Alternatively, attention priming could complement or conflict with the perceptual requirements of the task, in which case it was expected that global decisions should prime object discriminations between visually dissimilar items and local decisions should prime object discriminations between visually similar items, independently of category level.

In order to establish whether possible priming effects of global and local attention on object discrimination are due to either the spatial extent of attention or grouping/ parsing processes, Experiment 1 included three differentsized hierarchical stimuli (large, medium, and small; see Figure 1A). The sizes of the large and small hierarchical figures were adjusted so that the local level of the large hierarchical figure subtended the same visual angle as the global level of the small hierarchical figure. If global/ local priming effects on object discrimination were due to the allocation of attentional resources based on the size of the attentional window, there should be no differences in the effects of priming between the local level of the large figure and the global level of the small figure, because the size of the attentional window is the same for both levels. However, if global/local priming effects were due to grouping or parsing images into their global and local constituents, a different pattern of priming would be expected from the local level of the large figure and the global level of the small figure, because priming effects would be related to level-specific processes, not spatial extent.

\section{Method}

Participants. Sixty-one undergraduate students from Dalhousie University participated in the experiment to fulfill a course requirement. All had normal or corrected-to-normal vision and provided written, informed consent.

Stimuli. The hierarchical stimuli for the divided attention task were $2 \mathrm{~s}$ and $5 \mathrm{~s}$ made up of $8 \mathrm{~s}$, or $8 \mathrm{~s}$ made up of small $2 \mathrm{~s}$ and $5 \mathrm{~s}$ (see Figure 1A). There were three sizes of hierarchical stimuli. The large hierarchical stimuli were $8.7 \mathrm{~cm}$ tall $\times 6.2 \mathrm{~cm}$ wide at the global

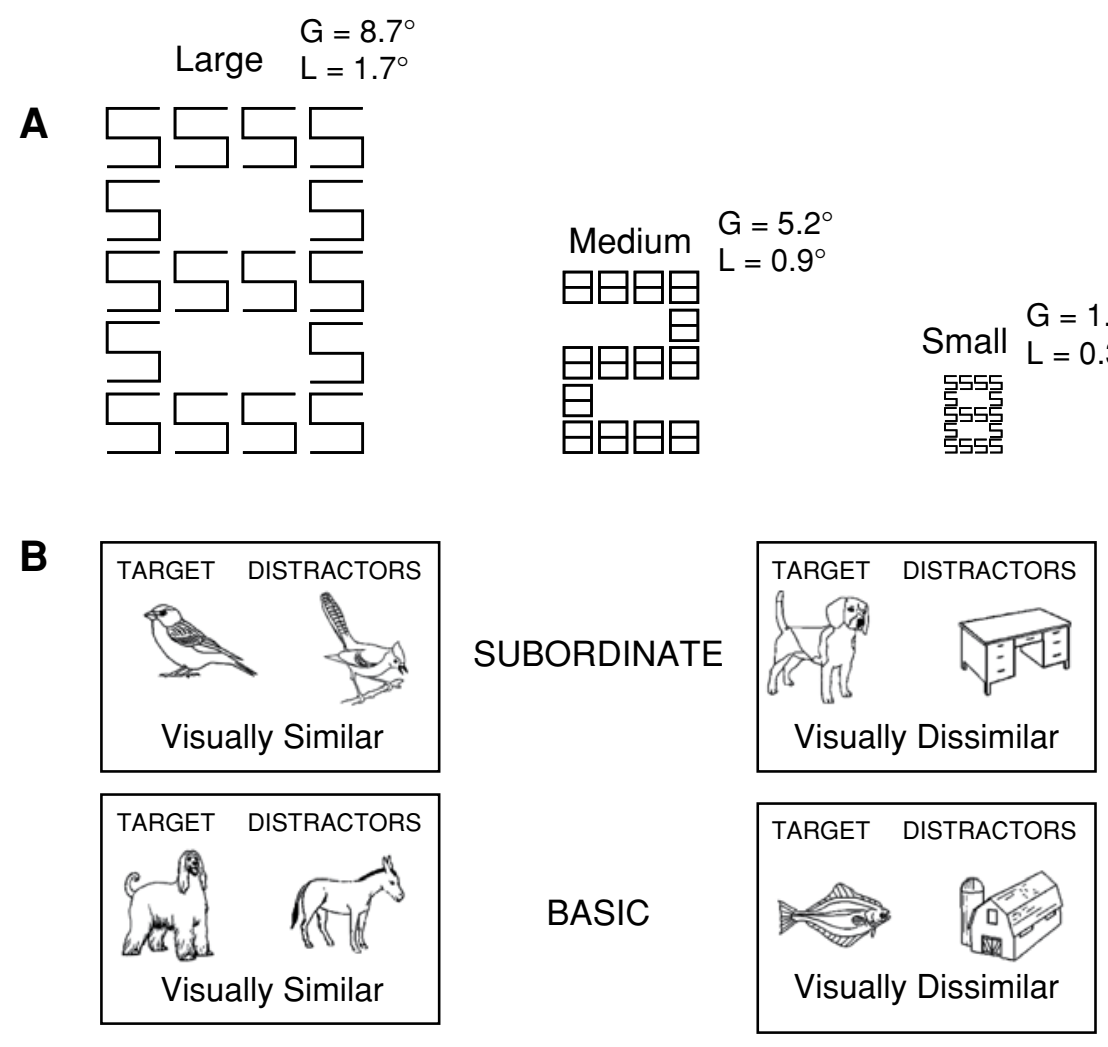

Figure 1. Examples of the stimuli used in the divided attention task and the object discrimination task in Experiment 1. (A) Hierarchical stimuli for the divided attention task. The approximate visual angles subtended at the global $(G)$ and local $(L)$ levels for each hierarchical figure are listed. (B) Examples of the stimuli used in the object discrimination task in Experiments 1 and 2. For each subordinate- and basic-level task, the targets and the distractors were either visually similar or visually dissimilar (see Appendix $C$ for a list of objects). Note-The global small stimulus subtends the same visual angle as the local large stimulus. 
level and $1.7 \mathrm{~cm}$ tall $\times 1.4 \mathrm{~cm}$ wide at the local level, subtending visual angles of approximately $8.7^{\circ}$ and $1.7^{\circ}$ at the global and local levels, respectively. The medium hierarchical stimuli were $5.2 \mathrm{~cm}$ tall $\times 3.7 \mathrm{~cm}$ wide at the global level and $0.9 \mathrm{~mm}$ tall $\times 0.74 \mathrm{~mm}$ wide at the local level, subtending visual angles of approximately $5.2^{\circ}$ and $0.9^{\circ}$. The small hierarchical stimuli were $1.7 \mathrm{~cm}$ tall $\times$ $1.4 \mathrm{~cm}$ wide at the global level and $0.3 \mathrm{~mm}$ tall $\times 0.25 \mathrm{~mm}$ wide at the local level, subtending visual angles of approximately $1.7^{\circ}$ and $0.3^{\circ}$.

In the object discrimination task, there were 176 black-and-white line drawings that consisted of 22 drawings each of finches, other types of birds, beagles, other types of dogs, mammals, and fish and 44 drawings of a variety of manmade objects (see Figure 1B and Appendix A). From each of the animal categories, 6 drawings were used in practice trials, and 12 manmade object drawings were also used in practice trials. The line drawings were taken from the Snodgrass and Vanderwart (1980) set, modified from children's coloring books, or created from photographic images, using Adobe Photoshop/ Illustrator. All the objects subtended an approximate visual angle of $5.2^{\circ}$. The stimuli from both tasks were presented on a white background, using custom-programmed software (MacInnes, 2000) on a Macintosh G3 computer.

Procedure. The participants first saw a fixation cross presented for $500 \mathrm{msec}$, followed by a global/local figure to which they responded with a keypress to identify the presence of a 2 or a 5 . This was followed by an intertrial interval of $1,000 \mathrm{msec}$, a second fixation cross $(500 \mathrm{msec})$, and an object to which they responded with one keypress if the object was a target and another if the object was a distractor. For both the divided attention task and the target discrimination task, the stimuli remained on the screen until the participant made a response or $3 \mathrm{sec}$ had passed. Half the participants responded to the divided attention task with their left hand and the object discrimination task with their right hand, and vice versa for the remaining participants. The divided attention task always preceded the object discrimination task, making a trial couplet. There was a block of trials for each of the object discrimination tasks. These were further divided into 3 blocks of trials, 1 for each of the three sizes of the hierarchical stimuli. Thus, there was a total of 12 blocks of test trials, each consisting of 32 trial couplets. It was decided to block the three sets of different-sized hierarchical figures because uncertainty about the size of the hierarchical stimulus might introduce an undesired variable. For example, Lamb and Robertson (1988) demonstrated that when participants were uncertain about the location of hierarchical figures, this eliminated local precedence. Random presentation of hierarchical figures varying in size would also introduce location uncertainty, since the figures would occupy variable regions of space.

Each test block was preceded by practice trials consisting of 12 trial couplets. In each block, the order of couplets was randomized. Half of the couplets in a block had global level targets, and half had local level targets. Half of the global level targets required a 2 response, and half a 5 response, as did the local-level targets. The divided attention task was the same in each block, but the object discrimination task differed. Instructions preceding each block indicated which set of images were targets and which set were distractors. As is depicted in Figure 1B, for visually similar subordinate categorizations, the targets were finches, and the distractors were other types of bird. For visually dissimilar subordinate decisions, the targets were beagles, and the distractors were manmade objects. For visually similar basic categorizations, the targets were $\operatorname{dogs}$, and the distractors were mammals, and for visually dissimilar basic categorizations, the targets were fish, and the distractors were manmade objects. The participants were shown pictures of beagles and finches to familiarize them with these target objects before proceeding with the experimental trials. These pictures were the same as those that had appeared in the practice trials. The presentation order of the four object discrimination tasks was randomized. For each object dis- crimination task, blocks of three different-sized hierarchical stimuli were presented in a Latin square order.

\section{Results}

Multifactorial repeated measures ANOVAs were performed on the reaction times (RTs) and percentages correct for the target discrimination task, with factors of attention level (global or local), category level (subordinate or basic), distractor type (similar or dissimilar), and size (large, medium, or small). For inclusion in the analysis, accuracy levels had to be above $50 \%$ in all of the cells in the factorial design. On the basis of this criterion, the data from 14 participants were excluded. Only couplets in which responses to both tasks were correct were analyzed. There was a robust effect of distractor type; object discriminations were significantly faster and more accurate when the distractors were dissimilar $[F(1,46)=91.53$, $p<.0001]$ than when the distractors were similar. This pattern of results occurred in all the subsequent analyses for Experiment 1 and will be omitted in the ensuing Results section. In all the reported results, outliers in the RT data were excluded on the basis of the method advocated by Van Selst and Jolicœur (1994) for evaluating outliers in designs with a small number of observations per cell. Analyses of RTs and percentages correct for the divided attention task are reported in Appendix B, since performance on this part of the trial couplets was not the focus of this study.

Reaction times. There was evidence that the degree of visual similarity between targets and distractors influenced performance on basic and subordinate object discriminations, on the basis of a two-way interaction between the factors of distractor type and category level $[F(1,46)=4.365, p=.04]$. Basic object discriminations $(M=688 \mathrm{msec})$ were faster than subordinate object discriminations $(M=733 \mathrm{msec})[t(1,281)=4.13, p<$ $.0001]$ when the distractors were visually similar. However, there was no reliable difference between category levels when the distractors were dissimilar $(t<1)$. There was also a two-way interaction between distractor type and size $[F(2,92)=4.45, p=.02]$. In the context of visually similar distractors, object discriminations made after responses to the medium-sized hierarchical figures $(M=$ $728 \mathrm{msec}$ ) were slower than object discriminations made after the large- and small-sized hierarchical figures (large, $697 \mathrm{msec}$; small, $707 \mathrm{msec}$ ). When the distractors were visually dissimilar, there was little difference in target discrimination times in terms of the size of the preceding hierarchical figures (large, $600 \mathrm{msec}$; medium, $586 \mathrm{msec}$; small, $594 \mathrm{msec}$ ).

Most important, there were a number of effects associated with global and local priming. First, the effects of global and local priming differed depending on the category level $[F(1,46)=8.02, p=.01]$ of the object discriminations. Second, the effects of global and local priming differed depending on the degree of visual similarity between the targets and the distractors $[F(1,46)=3.96, p=.05]$. The influence of global and local priming on object discrimina- 
tions is best understood by examining the combined effects of category level, distractor type, and attention level, since all three factors interacted $[F(1,46)=15.16, p=.001]$. Figure $2 \mathrm{~A}$ shows that subordinate object discriminations made in the context of visually similar distractors were faster when preceded by local decisions $(M=720 \mathrm{msec})$ than when preceded by global decisions $(M=750 \mathrm{msec})$ $[t(1,140)=2.75, p=.05]$. In contrast, basic-level object discriminations made in the context of visually similar distractors were faster when preceded by global decisions $(M=689 \mathrm{msec})$ than when preceded by local decisions $(M=702 \mathrm{msec})[t(1,140)=2.29, p=.01]$. Similarly, subordinate object discriminations made in the context of visually dissimilar distractors were faster when preceded by global decisions $(M=579 \mathrm{msec})$ than when preceded by local decisions $(M=604 \mathrm{msec})[t(1,140)=2.69, p=$ $.008]$. There was no reliable difference produced by global $(M=590 \mathrm{msec})$ and local $(M=605 \mathrm{msec})$ priming on basic-level object discriminations made in the context of visually dissimilar distractors $[t(1,140)=1.59, p=.11]$. Finally, there were no reliable effects of size associated with global and local priming.

Percentages correct. More correct responses were made for object discriminations when the distractors were dissimilar $(95 \%)$ than for object discriminations made when the distractors were similar $(91 \%)[F(1,46)=$ $36.08, p=.01]$. Again, this pattern of accuracy was found for all the subsequent analyses and will not be reported in the ensuing sections. There was a two-way interaction between the factors of size and category level $[F(2,94)=$ $4.99, p=.01]$. Performance was worse on subordinate object discriminations (92\%; basic, $94 \%$ ) after priming by small hierarchical figures and worse on basic object discriminations (92\%; subordinate, 94\%) after priming by medium hierarchical figures. There were no other significant effects. These measures failed to support any speed-accuracy trade-offs.

Object discriminations after priming by global (small) and local (large) hierarchical figures. To investigate the effects of global and local priming when the primes in these two conditions were the same physical size, a $2 \times 2 \times 2$ repeated measures ANOVA was performed on object discriminations, with factors of attention level (global small or local large), category level (subordinate or basic), and distractor type (similar or dissimilar). In support of the parsing hypothesis, there was a significant three-way interaction between the factors of attention level, category level, and distractor type $[F(1,46)=$ $4.745, p=.03]$. Figure $2 \mathrm{~B}$ shows a similar pattern of priming in response to global and local decisions, even though the primes subtend the same visual angles. Analysis of simple effects showed that after priming by global decisions, basic object discriminations were faster than subordinate object discriminations $[t(1,93)=2.53, p=$

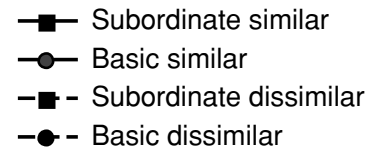

A

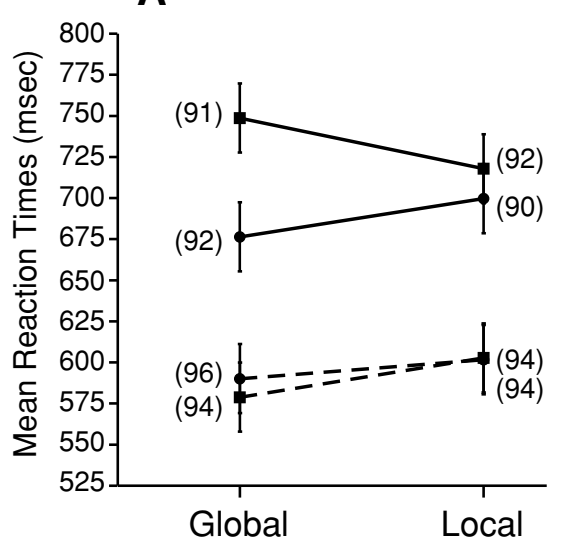

Attention Prime Level
B

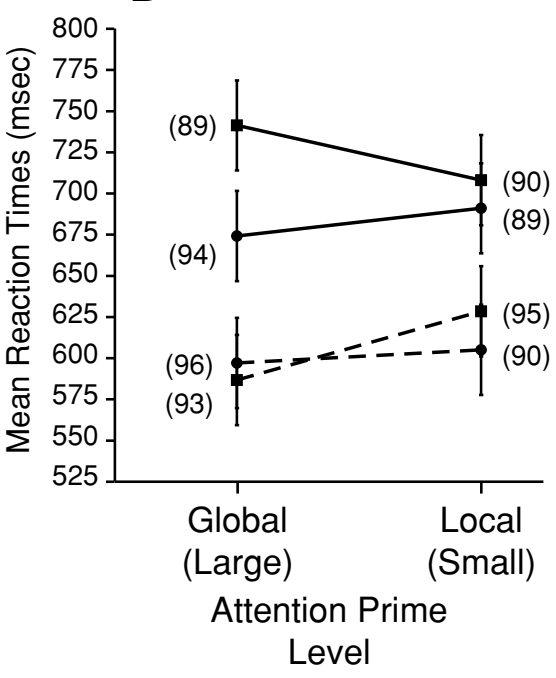

Figure 2. (A) Mean reaction times to the object discrimination task after priming by a global/local divided attention task collapsed across all sizes of the hierarchical primes. (B) Mean reaction times to the object discrimination task after priming of attention with either the global level of the small hierarchical figure or the local level of the large hierarchical figure. Both attention primes subtended the same visual angle. The numbers in parentheses are the percentages correct for each condition, and the bars denote within-subjects confidence intervals (Loftus \& Masson, 1994). 
.02]. However, there was no reliable difference between subordinate and basic discriminations after priming by local decisions $(t<1)$.

Analysis of the accuracy data demonstrated a main effect of category level $[F(1,46)=3.91, p=.05]$, with more correct responses to basic object discriminations (94\%) than to subordinate object discriminations (92\%). The effects of global (small) and local (large) priming also differed depending on the category level of the target $[F(1,46)=4.94, p=.03]$, with more correct responses made to basic object discriminations preceded by global decisions (95\%) than to those preceded by local decisions $(92 \%)$ and little difference in subordinate object discriminations after priming by either global or local decisions (91\% and $92 \%$, respectively). This interaction provides further support for the parsing hypothesis and failed to support any speed-accuracy trade-offs.

\section{Discussion}

The evidence reported supports the notion that global and local attention selectively modulated the subsequent object discriminations, similar to evidence found with spatial scale (Archambault et al., 2000; Schyns \& Oliva, 1999). Priming by local-level decisions facilitated subordinate object discriminations, and priming by global decisions facilitated basic object discriminations. Priming by global decisions also facilitated subordinate object discriminations when the distractors were visually dissimilar. Shifts between global and local levels of attention may act to facilitate the selection of coarse- or fine-grained information from the stimulus. It is possible that when the visual system is primed to process coarse-grained aspects of visual stimuli, this benefits discriminations between visually dissimilar objects and reduces the efficiency of discriminations between visually similar objects. In contrast, when the visual system is primed to process fine details of visual stimuli, this benefits discriminations between visually similar objects and reduces the efficiency of discriminations between visually dissimilar objects.

The second question addressed in this experiment was whether priming by hierarchical figures was mediated by a parsing mechanism or by the size of the attentional window. Evidence was found that supported the role of a parsing mechanism. A comparison was made between the priming effects of two stimuli that subtended the same visual angle but belonged to different levels of a hierarchical figurenamely, the local level of the large hierarchical stimulus versus the global level of the small hierarchical stimulus. The results showed that there was a difference in the priming effects of these two stimuli. In particular, basic-level object discriminations made in the context of visually similar distractors were faster when preceded by the global level of the small hierarchical stimulus, in comparison with subordinate object discriminations, which were faster when preceded by the local level of the large stimulus. In contrast, there was no reliable difference between subordinate and basic object discriminations when preceded by the local level of the large hierarchical stimulus. If the priming effects of global and local attention on the object recognition task were due only to the size of the attentional window, there should have been no difference between the effects of these two attentional primes, since they were the same size.

A strong prediction of the parsing hypothesis would be that the size of the hierarchical figures would not influence global- and local-priming effects on target discrimination. Indeed, the size of the hierarchical stimuli did not influence the effects of global- and local-level priming on object discrimination. However, the overall size of the hierarchical figures did influence the speed at which the participants responded to target objects. Object discriminations made in the context of visually similar distractors were slower when preceded by a medium-sized hierarchical figure than when they were preceded by small- or large-sized hierarchical figures, regardless of whether the prime was at the local or the global level. That is, a prime that was the same size as the target object slowed target discrimination in the context of visually similar distractors. This would suggest that some element of attention priming was related to the size of the attentional window. This finding is not unprecedented. Other researchers have also found evidence supporting the involvement of both parsing and spatial extent mechanisms in the processing of global and local figures (Lamb \& Yund, 2000; Robertson et al., 1993). The interesting question here is how these two mechanisms contributed to the performance of the object discrimination task.

Research conducted by Kim et al. (1999) may shed light on this question. Kim et al. were interested in finding out whether parsing objects into their global and local forms was based on the absolute size of the stimulus or was relative to the physical attributes of the stimulus. They presented participants with different-sized hierarchical stimuli. The global level of the small hierarchical stimulus was the same size as the local level of the large hierarchical stimulus. They examined level repetition effects and size repetition effects and found that level repetition effects were influenced by the overall size of the hierarchical forms. Their results suggested that level repetition effects were not independent of the absolute size of the hierarchical forms. Kim et al. argued that their data could be explained by a dual-frequency filtering mechanism that first selects the absolute frequency range in a stimulus and then selects the relative frequencies within the range (see also Robertson \& Ivry, 2000). Either of these filtering operations could influence object discriminations on subsequent trials.

The different pattern of priming found for the large-, medium-, and small-sized primes in this experiment might be explained by one mechanism that adjusts the attentional window to the overall sizes of the hierarchical forms and objects and a second mechanism that parses object features into global and local forms relative to the size of the attentional window. The fact that people are able to recognize objects in many different formats, such as black-and-white or colored photographs and illustrations, line drawings, cartoons, and variously degraded images, supports the notion that numerous properties of a visual stimulus are diagnostic for its identity. The hierarchical 
primes may bias the visual system to process one set of perceptual cues, instead of another set of perceptual cues. In effect, changing the sizes of the hierarchical figures modulates attentional processes and biases the visual system in favor of different perceptual cues.

There were also effects that did not involve attention priming. Object discriminations made in the context of visually similar distractors were slower than object discriminations made in the context of visually dissimilar distractors, demonstrating that it is easier to distinguish between objects that are visually dissimilar than between objects that are visually dissimilar. A difference was also found between subordinate object discriminations and basic object discriminations made in the context of visually similar versus visually dissimilar distractors. When the distractors were visually similar to the targets, basic object discriminations were faster than subordinate object discriminations. However, when the distractors were visually dissimilar to the targets, no reliable difference was found between subordinate and basic object discriminations. This result suggests that differences in performance between subordinate and basic object discriminations do depend on the degree of visual similarity between the target and the distractor objects in a visual task. That is, visual similarity between members belonging to the same category or between members belonging to different categories can influence the performance of category judgments (Jolicœur et al., 1984; Lloyd-Jones \& Humphreys, 1997a, 1997b; Murphy \& Brownell, 1985; Rosch et al., 1976; Snodgrass \& McCullough, 1986).

Given that visual similarity is a factor that strongly affects categorization, one could argue that the factor of category level is redundant. However, when images of common objects are used, it is difficult to know how much prior knowledge, as compared with more input-driven processes, will influence performance. It is entirely possible that with more realistic objects, prior knowledge could affect whether global or local levels of processing are instigated. There is evidence that conceptual knowledge of objects impacts perceptual processes. In a series of studies, Boucart and her colleagues (Boucart \& Humphreys, 1992; Boucart, Humphreys, \& Lorenceau, 1995; Humphreys \& Boucart, 1997; see also Murray \& Jones, 2002) found that semantic information affected judgments about the physical properties of objects, such as their global shape (oval or round), surface texture, motion, color, and the orientation of embedded lines. If conceptual knowledge can influence perceptual processes, we wanted to allow for the possibility that the allocation of attentional resources at either the global or the local level could be mediated by prior knowledge of the semantic properties of the target objects. For example, when discriminating a particular breed of dog, such as a beagle, previous semantic knowledge suggests that breeds of dogs are highly visually similar, and processing fine visual details (local level of attention) is more likely to produce accurate detections. On the basis of semantic knowledge, a level of attention may be selected prior to the task, and the effects of priming attention with the global/local task could facilitate or interfere with this prior selection. Therefore, local priming could have facilitated the detection of subordinate objects (finches and beagles), regardless of the visual similarity of the distractor objects. But the evidence from this experiment suggests that the effects of global and local priming were related more to the degree of visual similarity between target objects and distractors than to the category level at which the objects were labeled.

In conclusion, the results of this experiment indicate that global and local processing modulated the usage of perceptual information in the object discrimination task. When visually similar objects were discriminated between at a subordinate level of categorization, perceptual information supported by local processing was more important than perceptual information supported by global processing. Conversely, when visually dissimilar objects were discriminated between and basic-level decisions were made, perceptual information supported by global processing was more important than perceptual information supported by local processing. Experiment 1 also provided evidence that predominantly supported the parsing mechanism. Global and local primes subtending the same visual angles produced different effects in the object discrimination task. However, effects of size were also observed on the object discrimination task, so there is some support for both mechanisms. In Experiment 2, we further explored the role of the size of the attentional window on object discrimination.

\section{EXPERIMENT 2}

In Experiment 1, it was argued that the pattern of attention priming on the object target discrimination task was mainly due to parsing operations. However, there was some evidence that the spatial extent of attention influenced the discrimination of objects, since the overall size of the hierarchical primes differentially affected object discriminations when the objects were visually similar. The purpose of Experiment 2 was to test whether resizing the attentional window without a parsing component would influence performance on object discriminations.

The main evidence for the parsing mechanism in Experiment 1 was the fact that priming by the local level of the large hierarchical figure differed from priming by the global level of the small hierarchical figure, even though both of them subtended the same visual angle. However, the large and small hierarchical figures subtended different visual angles as a whole. Effectively, the overall distribution of attention would differ for each of these figures. Thus, the different patterns of priming observed for large and small hierarchical figures could have reflected a difference in the distribution of attention based on the overall size of the hierarchical figures.

In Experiment 2, the target discrimination task was the same as that used in Experiment 1, but the attentionpriming task was modified. The global and local levels of the hierarchical figures were separated into individual (nonhierarchical) digits (see Figure 3). For example, the large hierarchical prime was separated into individual dig- 


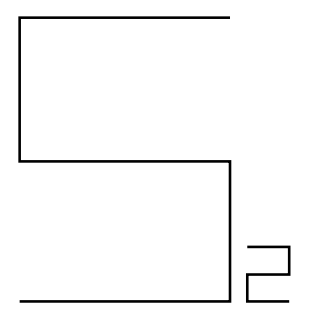

A) Large Digit Pair
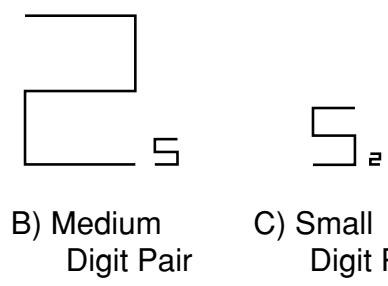

C) Small Digit Pair

Figure 3. The digits used in the attention-priming task for Experiment 2 . Stimuli were constructed by separating out the global and the local levels of the hierarchical figures in Experiment 1 at each of the three sizes (A, large; $B$, medium; and $C$, small). The digits subtended the same visual angles as the global (big digit) and local (little digit) elements of the hierarchical figures.

its subtending visual angles of $8.7^{\circ}$ for the global level and $1.7^{\circ}$ for the local level. The task of the participant in the attention-priming condition was to indicate whether the digit was a 2 or a 5 . In effect, the digits act in a similar fashion to the rectangular cues used by Robertson et al. (1993) and Lamb and Yund (2000) in their investigations into the effects of cuing the region of space covered by global or local targets.

There would be support for the attentional window hypothesis if similar priming effects on the target discrimination task were observed when the target was primed by separate digits subtending the same visual angles as the global and local levels of the hierarchical figures. On the other hand, there would be support for the parsing hypothesis if there were no reliable effects on the target discrimination task when the target was primed by separate digits or the patterns of priming differed markedly from those observed in Experiment 2. In addition, there should be no reliable difference in the priming effects for digits subtending the same visual angle.

\footnotetext{
Method

Participants. Sixty-four undergraduate students from Dalhousie University participated in the experiment to fulfil a course requirement. All had normal or corrected-to-normal vision and provided written, informed consent.

Stimuli. The stimuli for the attention task were the digits 2 and 5 . The stimuli were constructed by separating out the global and local levels of the hierarchical figures in Experiment 1, producing three different-sized digit pairs, as depicted in Figure 3. The digit pairs corresponded in size to the global and local components of the hierarchical figures presented in Experiment 1 (see Figure 1A for examples). The digit pairs taken from the large hierarchical figure subtended visual angles of approximately $8.7^{\circ}$ and $1.7^{\circ}$. The digit pairs taken from the medium hierarchical figure subtended visual angles of approximately $5.2^{\circ}$ and $0.9^{\circ}$, and the digit pairs taken from the small hierarchical figure subtended visual angles of approximately $1.7^{\circ}$ and $0.3^{\circ}$. The same 176 black-and-white line drawings as those in Experiment 1 were used in the target discrimination task (see Figure 1B and Appendix A). All the objects subtended approximate visual angles of $5.2^{\circ}$. The stimuli from both tasks were presented on a white background, using custom-programmed software (MacInnes, 2000) on a Macintosh G3 computer.
}

Procedure. The procedure was the same as that described in Experiment 1 , except that in the attention-priming task, single digits were presented on the computer screen, to which the participants responded with either a 2 or a 5 keypress. The digit pairs corresponding in size to the global and local levels of the hierarchical figures were presented in three separate blocks for each of the digit pair sizes (large, medium, and small).

\section{Results}

Multifactorial repeated measures ANOVAs were performed on the RTs and percentages correct for the target discrimination task. The factors were spatial extent (global element or local element), size of digit pairs (large, medium, or small), distractor type (visually similar or visually dissimilar) and category level (subordinate or basic). Data were included in the RT analysis only if two consecutive responses in a couplet were correct. Seventeen participants were excluded, since they achieved less than $50 \%$ correct in one or more of the cells in the factorial design. As in Experiment 1, target object discriminations were significantly faster and more accurate when the distractors were dissimilar $[F(1,47)=197.44, p<.0001]$ than when the distractors were similar. This pattern of results occurred in all the subsequent analyses for Experiment 2 and will be omitted in the ensuing Results sections. The analysis of RTs and percentages correct for the divided attention task is reported in Appendix C.

Reaction times. There was evidence that the degree of visual similarity between targets and distractors influenced performance on basic and subordinate object discriminations, on the basis of a two-way interaction between the factors of distractor type and category level $[F(1,47)=8.52, p=.01]$. Basic object discriminations $(M=680 \mathrm{msec})$ were faster than subordinate object discriminations $(M=705 \mathrm{msec})[t(1,287)=3.03, p=$ $.003]$ when the distractors were visually similar. In the case of visually dissimilar distractors, the opposite pattern was observed; subordinate object discriminations $(M=$ $561 \mathrm{msec}$ ) were faster than basic object discriminations $(M=578 \mathrm{msec})[t(1,287)=3.20, p=.001]$.

In support of the attentional window hypothesis, priming by global and local nonhierarchical elements was influenced by the category level of the targets $[F(1,47)=$ $4.87, p=.03]$. Subordinate object discriminations were faster when preceded by local nonhierarchical elements $(M=628 \mathrm{msec})$ than when preceded by global nonhierarchical elements $(M=638 \mathrm{msec})[t(1,287)=1.93, p=$ $.05]$, whereas there was no reliable difference in basic object discriminations after global nonhierarchical $(M=$ $627 \mathrm{msec})$ and local nonhierarchical $(M=631 \mathrm{msec})$ priming $(t=1)$. From Figure 4A, it appears that this effect was due mainly to differences in object discrimination times when the distractors were visually similar.

Furthermore, unlike in the data from Experiment 1, attention priming was influenced by the overall size of the digit pairs $[F(2,94)=3.33, p=.04]$. Object discriminations made after small digit pairs were faster when preceded by the local nonhierarchical elements $(M=627 \mathrm{msec})$ than when preceded by global nonhierarchical elements $(M=$ $644 \mathrm{msec})[t(1,91)=2.14, p=.03]$. There were no reli- 


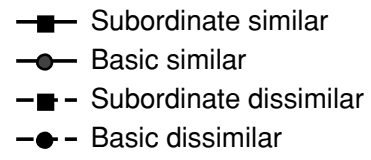

A

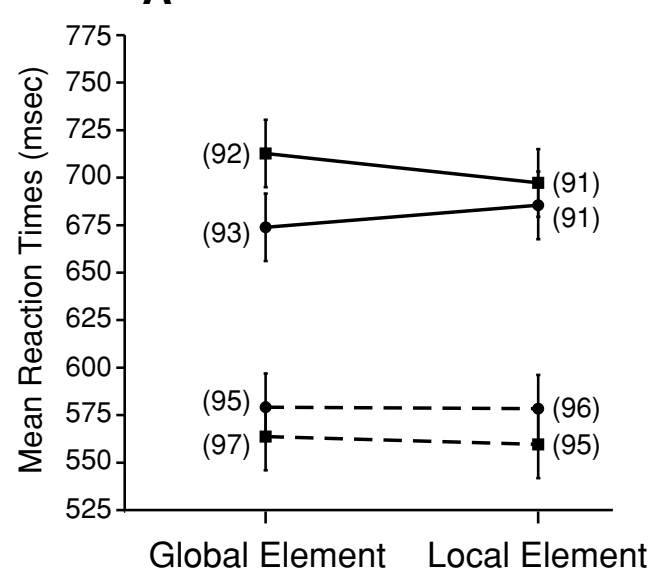

Attention Prime
B

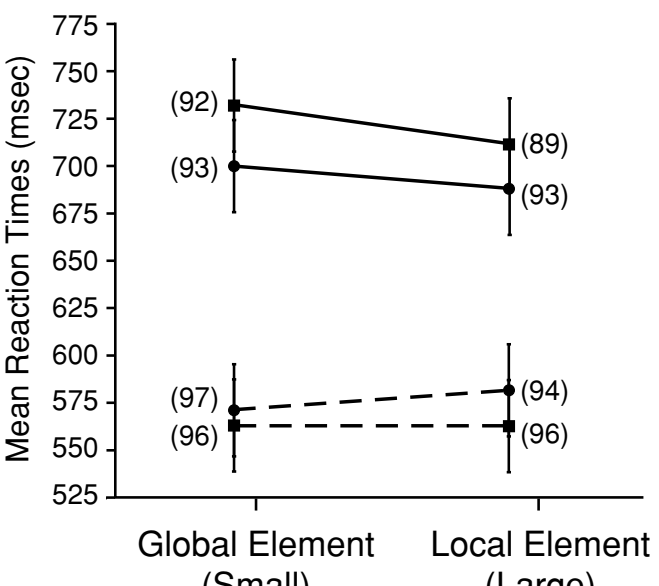

(Small) Level Level

\begin{abstract}
Figure 4. (A) Mean reaction times to the object discrimination task after priming by variously sized digits. The data are collapsed across three digit pairs (large, medium, and small; see Figure 3). The "global element" and "local element" labels refer to the origins of the attention primes. Global element primes were the digits 2 and 5 that subtended the same visual angles as the global levels of the hierarchical primes in Experiment 2. Local element primes were the digits that subtended the same visual angles as the local levels of the hierarchical primes in Experiment 2. (B) Mean reaction times to the object discrimination task after priming by the digits 2 and 5 , which subtended the same visual angle and were constructed from the global element of the small hierarchical figure (global small) and the local element of the large hierarchical figure (local large) used in Experiment 2. The numbers in parentheses are the percentages correct for each condition, and the bars denote within-subjects confidence intervals (Loftus \& Masson, 1994).
\end{abstract}

able differences in object discrimination times after medium or large digit pairs $(t<1)$. The influence of size on attention priming was further mediated by the degree of visual similarity between the targets and the distractors $[F(2,94)=7.99, p=.001]$. As is evident in Figure 5, object discriminations made in the context of visually similar distractors were faster when preceded by global nonhierarchical elements $(M=673 \mathrm{msec})$ than when preceded by local nonhierarchical elements from the large digit pair $(M=$ $699 \mathrm{msec})[t(1,95)=2.67, p=.01]$. On the other hand, object discriminations preceded by local nonhierarchical elements $(M=687 \mathrm{msec})$ were faster than object discriminations preceded by global nonhierarchical elements $(M=$ $715 \mathrm{msec})[t(1,95)=2.51, p=.01]$ from the small digit pair. Effectively, when the overall size of the attentional window was large, object discriminations were faster when primed by the bigger of the two digits. But when the overall size of the attentional window was small, object discriminations were faster when primed by the smaller of the two digits. There was no significant difference between object discriminations made in the context of visually dissimilar distractors preceded by global and local nonhierarchical elements at any of the three digit pair sizes.
Percentages correct. More correct responses were made for object discriminations when the distractors were dissimilar (96\%) than for object discriminations when the distractors were similar $(92 \%)[F(1,47)=29.5, p<$ $.0001]$. Accuracy was also influenced by attention priming $[F(1,47)=3.94, p=.05]$, with more correct responses being made for object discriminations preceded by global nonhierarchical elements (94\%) than for object discriminations preceded by local nonhierarchical elements $(93 \%)$. There were no other significant effects. These measures failed to support any speed-accuracy trade-offs.

Object discriminations after priming by the global element (small digit pair) and the local element (large digit pair). To test the attentional window hypothesis, the effects of priming by the global element from the small digit pair (Figure 3C) and the local element from the large digit pair (Figure 3A) were compared, since these two primes were of the same physical size. If the attentional window hypothesis is supported, there should be differences in the effects of these two primes on the object discrimination responses. RTs to targets differed depending on the degree of visual similarity between the targets and the distractors $[F(1,47)=212.27, p<.0001]$. No other 


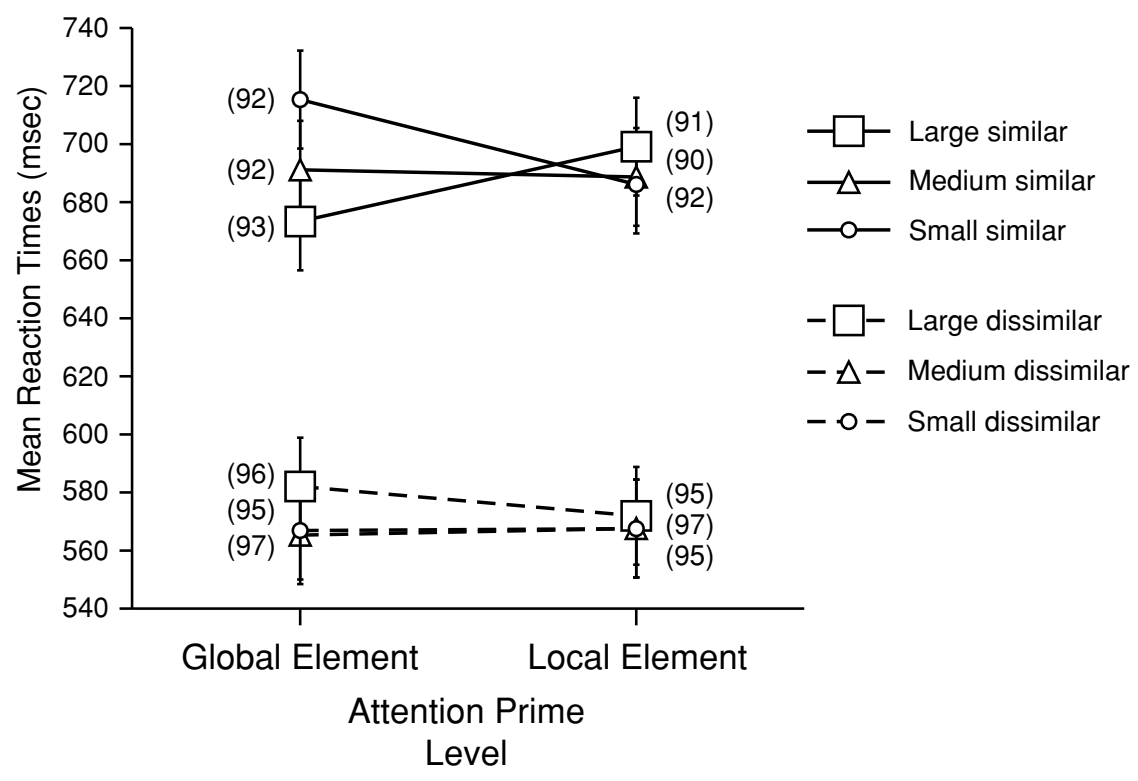

Figure 5. Mean reaction times to basic and subordinate object discriminations made in the context of visually similar or visually dissimilar distractors after priming by single digits that varied in size. The attention primes were the digits 2 and 5 , constructed by separating out the global and local elements of the large, medium, and small hierarchical figures used in Experiment 2. The numbers in parentheses are the percentages correct for each condition, and the bars denote within-subjects confidence intervals (Loftus \& Masson, 1994).

effects were significant. From Figure 4B, it can be seen that attention priming by separate digits subtending the same visual angle produced similar priming effects on the object discrimination tasks.

In the accuracy of object discriminations, there was a main effect of spatial extent $[F(1,47)=5.75, p=.02]$, with more correct responses to object discriminations made after priming by the global nonhierarchical element of the small digit pair (95\%) than after priming by the local nonhierarchical element of the large digit pair (93\%). These measures failed to support any speed-accuracy trade-offs.

\section{Discussion}

In Experiment 2, we investigated whether the size of the attentional window, in the absence of any hierarchy to the primes, would influence object discriminations. Subordinate object discriminations were faster when preceded by local element decisions than when preceded by global element decisions for small- and medium-sized primes. In contrast, basic object discriminations were faster when preceded by global element decisions than when preceded by local element decisions for large- and medium-sized primes. However, there were a number of differences in the effects of attention priming between Experiments 1 and 2 , suggesting that the attentional window hypothesis could not account for all the priming effects observed using hierarchical figures in Experiment 1.

In particular, different priming effects were observed in Experiment 1 for stimuli that subtended the same visual angle but belonged to different levels of a hierarchical figure (global vs. local). In Experiment 2, priming effects of the global and local elements subtending the same size, but without any hierarchy, did not produce any reliable differences in target discrimination times. Also, priming by single digits did not produce effects on object discriminations when the distractors were visually dissimilar. In contrast, subordinate decisions made in the context of visually dissimilar distractors were faster when primed by the global level of hierarchical figures. Finally, in Experiment 1, the size of the hierarchical primes did not influence global and local priming effects. However, when these global and local elements were presented as single digits, there were marked differences in the priming effects of these nonhierarchical elements, depending on whether they belonged to the large digit pair or the small digit pair. In the large digit pair, object discriminations made in the context of visually similar distractors were faster when preceded by global elements than when preceded by local elements. The opposite pattern was found for object discriminations primed by the small digit pair; object discriminations preceded by local elements were faster than those preceded by global elements.

Interestingly, object discriminations made in the context of visually similar distractors were faster when preceded by digits subtending either the smallest or the largest visual angles, relative to the visual angle subtended by the objects. Subordinate and basic object discriminations preceded by the global nonhierarchical element of the large digit group $\left(8.7^{\circ}\right)$ were faster than object discriminations preceded by the local nonhierarchical element $\left(1.7^{\circ}\right)$ $[t(1,47)=2.85, p=.006]$. In contrast, subordinate and basic object discriminations preceded by the local non- 
hierarchical element of the small digit group $\left(0.9^{\circ}\right)$ were faster than object discriminations preceded by the global nonhierarchical element $\left(1.7^{\circ}\right)[t(1,47)=2.39, p=.02]$. These results would suggest that large increases or decreases in the size of the attentional window benefited more demanding object discriminations.

Fiser, Subramaniam, and Biederman (2001) investigated the effects of size variation on object discrimination and found that the visual system was sensitive to these variations and could "tune into" particular size ranges. They used a rapid serial visual presentation (RSVP) task in which 40 gray-level object images were presented. The participants' task was to indicate with a keypress the presence of a target object in the sequence. Two types of sequences were used: a homogenous sequence, in which all the images were the same size, and a heterogeneous sequence, in which one of the images (half of the time a target) was a different size from all the other images. They found that an advantage in correct identifications for large targets, in comparison with small targets, in a single sequential presentation task was eliminated in the homogeneous RSVP task. They proposed that the size of the images preceding and following the target influenced recognition. Their results and the results of this experiment indicate that attention to particular regions in space influences visual processing.

Fiser et al. (2001) also found that large targets embedded in a sequence of small images were detected more accurately than small targets embedded in a sequence of large targets. They speculated that this asymmetry in performance was due to adaptation mechanisms that show faster adaptation to abrupt increases than to abrupt decreases, similar to that found in light intensity adaptation in sensory cells. A similar finding was observed in the present experiment when object discriminations were primed with separate digits. Object discriminations were faster when preceded by small local elements than when preceded by small global elements. However, object discriminations were also faster when preceded by large global elements than when preceded by large local elements, arguing against an adaptation mechanism that results in asymmetrical performances. In Experiment 2, object discriminations benefited from both a large increase and a large decrease in size between the prime and the object.

The difference in results between Fiser et al.'s (2001) study and this experiment may be due to the use of different dependent measures. Fiser et al. used accuracy as a measure for their effects, and this experiment focused on RTs. There were also significant methodological differences. The sizes of the stimuli preceding the target in the RSVP task did not vary, whereas the sizes of the stimuli preceding the targets in the object discrimination task in this experiment switched randomly between two sizes.

In conclusion, Experiment 2 demonstrated that resizing the attentional window influenced object discriminations. In general, subordinate object discriminations were faster when preceded by local elements than when preceded by global elements, and basic object discriminations were faster when preceded by global elements than when preceded by local elements. In addition, object discriminations benefited when the attentional window was at the largest or smallest size, relative to the target objects. However, the attentional window hypothesis could not account entirely for the priming effects of hierarchical figures on object discriminations.

\section{GENERAL DISCUSSION}

The objective of the present experiments was to investigate whether global and local processing played a role in a more realistic object recognition task. Evidence was found that supported a role for global and local processing in the recognition of real-world objects. Local-level priming facilitated subordinate object discriminations made in the context of visually similar distractors. The effects of global priming were less consistent but, most often, associated with basic object discriminations or object discriminations made in the context of visually dissimilar distractors. This suggests that shifts between global and local levels of attention facilitate the selection of coarseor fine-grained information from the stimulus. When visually similar objects are categorized, the attentional bias is toward fine-grained information selected at the local level; conversely, when visually dissimilar objects are categorized, the attentional bias is toward coarse-grained information selected at the global level.

According to Han et al. (1999a), the perceptual attributes of objects are separated into global and local processing streams that operate in parallel and compete with each other to produce a response output. Subordinate object discriminations made in the context of visually similar objects were faster when preceded by local decisions. This condition is most comparable to strict definitions of subordinate categorization. The target objects and distractor objects belonged to the same basic-level category and shared many features in common. It would require processing the finer details of the object's shape to discriminate between these visually similar objects. In these circumstances, the perceptual attributes related to fine details of the object's shape that are represented in local stream were more likely to win the competition than were coarse-grained perceptual attributes represented in the global stream.

In contrast, global and local priming effects on basic object discriminations were more variable. Perhaps, for basic-level categorizations, there are more avenues available for correct identification. Archambault et al. (2000) speculated that the reason basic-level categorizations were more robust to changes in the size of the stimuli was because perceptual cues conveyed by either low or high spatial frequencies were equally diagnostic for basic-level identifications. A recent study by Collin and McMullen (2005) showed that, indeed, subordinate-level identifications rely on high spatial frequencies much more than basic-level identifications do. The differences in priming effects for basic-level object discriminations could reflect the flexibility of basic-level categorization processes in 
the usage of perceptual cues. For example, detailed information, such as the shape of a dog's paws or the shape of deer's hooves, could allow one to distinguish between a dog and a deer equally as well as would gross differences in body shape. The same could be said for subordinate object discriminations when they are made in the context of visually dissimilar distractors. Discriminating a beagle from a gun could be done equally well with detailed information, such as the shape of the nose, or from coarse-scale information, such as body shape. It is also possible that the strategy used by the participants in the visually dissimilar conditions was to discriminate the target object from the distractors on the basis of an animate versus inanimate distinction (i.e., superordinate-level decisions). If this is the case, the results suggest that superordinate-level decisions also favor global processing, but only when the target objects themselves are highly visually similar. When the target objects present more variability (fish vs. manmade objects), there was no difference in the priming effects of global and local decisions.

In this study, we also investigated whether global and local priming effects on object discrimination were mediated by two possible mechanisms - one based on the spatial extent of attention and the second on grouping/parsing processes. In support of the parsing hypothesis, different priming effects were observed for stimuli that subtended the same visual angle but occupied different levels in a hierarchical figure. In contrast, when the hierarchical figures were replaced by separate digits that subtended the same visual angles, no reliable differences were found in their priming effects on the object discrimination task. This would suggest that the priming effects of the global and local decisions were not related to the spatial extent of attention but to grouping and parsing processes acting on the hierarchical levels of the object representations.

However, the attentional window hypothesis could not be completely discounted, since there was evidence showing that the spatial extent of attention can influence visual processing. In Experiment 2, we found that when objects that were visually similar were discriminated between, a smaller attentional window favored perceptual attributes represented in the local processing stream, and a larger attentional window favored perceptual attributes represented in the global stream. Furthermore, in Experiment 1, it was found that the overall size of the hierarchical figures had an impact on object discriminations, regardless of the hierarchical level of the attention prime.

The results of the present experiments suggest that both parsing and spatial extent have a role to play in object recognition. These two processes could serve different functions in object recognition. Modulations in the size of the attentional window may increase the efficiency with which perceptual attributes are selected for further processing. Parsing processes preserve the hierarchical information contained in a visual stimulus and could facilitate the access of structural descriptions stored in memory. Han et al. (1999a) and Edelman and Intrator (2003) have proposed that the discrimination of parts or features of objects could occur with respect to hierarchical levels of the representation. For example, legs may be represented as parts relative to the body, and paws may be represented as parts relative to the legs. The spatial extent of attention may be adjusted by top-down processes to accommodate the processing of perceptual attributes necessary for the formation of an object representation at a level in the hierarchy that is appropriate for the object recognition task. Parsing processes may be used to further separate these perceptual attributes into parallel global and local processing streams. Information in these two processing streams could then compete to produce a response. For example, if the task is to discriminate an image of a dog, the attentional window may be set to cover the region of the image in which the shape of the dog appears. Parsing processes then separate the perceptual attributes of the shape into global and local processing streams. If the object recognition task requires discriminating the dog from other dogs, the perceptual attributes relating the fine details of the object's shape, which are represented in local stream, will likely win the competition. If the object recognition task requires discriminating the dog from manmade objects, perceptual attributes relating coarse shape information, which are represented in the global stream, will likely win the competition.

\section{Implications for Theories of Object Recognition}

Viewpoint-independent theories of object recognition hold that representations of objects are stored in memory as structural descriptions consisting of three-dimensional volumetric components and their configurations (Biederman, 1987, 1995; Marr, 1982). A major advantage of viewpoint-independent theories of object recognition proposed by Marr and Biederman is their ability to explicitly code for the parts of objects. The importance of object parts has been demonstrated by a number of researchers (Biederman \& Cooper, 1991; Hoffman \& Richards, 1984; Hoffman \& Singh, 1997; Tversky \& Hemenway, 1984). It has been argued that part decomposition is an important factor in object categorization (Edelman \& Intrator, 2003; Hummel, 2000; Tarr \& Bülthoff, 1995; Tarr \& Kriegman, 2001). For example, the discrimination of head and legs may help to identify an object as an animal, as opposed to a vehicle.

Marr (1982) proposed that object representations are hierarchical and modular in their organization. At the top of the hierarchy, there is a representation of the gross properties of an object, such as its orientation and size. This representation can be further decomposed on the basis of component axes into additional representations, each containing unique shape information that varies in its level of structural detail about an object. Progression through the hierarchy of modular representations of an object leads to access to more specific information about an object's shape. The further the progression through the hierarchy, the more distinctive objects become from each other.

Global and local processing may play a role in decomposing objects into their constituent parts or by modulating the selection of coarse- or fine-scale visual information at the input stage of object recognition. Global 
processing captures coarse-scale visual information (or large-scale parts) relevant for generating input representations that match to modular components of the structural description at the upper levels of the hierarchy. Similarly, local processing captures fine-scale visual information (or small-scale parts) relevant for generating input representations that match components at the lower levels of the hierarchy.

It is difficult to conceive of a role for global and local processing in line with view-dependent theories of object recognition. View-dependent theories of object recognition propose that a number of viewpoint-specific representations are held in memory. Object recognition is based on computing the similarity between the input representation and the stored view-based representations (Bülthoff \& Edelman, 1992; Edelman \& Duvdevani-Bar, 1997; Tarr \& Bülthoff, 1995; Tarr \& Pinker, 1989, 1990; for a review, see Vecera, 1998). The main thrust of view-dependent theories is to account for the recognition of objects when seen from unfamiliar vantage points without recourse to explicitly representing spatially distinct parts. Effectively, these theories make no distinction between coarse- and fine-scale information as it relates to the visual structure of objects.

Recently, however, Edelman and Intrator (2003) have proposed a computational model (the chorus of fragments model) that is viewpoint dependent and incorporates the notion of a hierarchy of structure. They proposed that different hierarchical levels of representation could be controlled via attentional processes that can steer attention to specific locations in an image and control the spatial resolution of the window of attention at that location. In effect, at the global level, the spatial extent and low resolution of the window of attention would allow for the discrimination of coarse details of an object. At the local level, the spatial extent and high resolution of the window of attention would allow for the discrimination of the fine details of an object.

In conclusion, evidence was found to support a role for global and local processing in the recognition of realworld objects. Global priming was beneficial for basiclevel target discriminations and for discriminations of visually dissimilar objects. Local priming was beneficial for subordinate-level target discriminations when objects were visually similar. It was proposed that global and local processing aids the selection of perceptual attributes such as coarse-scale or fine-scale features of the object that are diagnostic for recognition. Furthermore, the selection of perceptual attributes for discriminating between objects was found to be based on two mechanisms: spatial extent and grouping/parsing operations.

\section{REFERENCES}

Archambault, A., Gosselin, F., \& Schyns, P. G. (2000). A natural bias for the basic level? In L. R. Gleitman \& A. K. Joshi (Eds.), Proceedings of the twenty-second annual conference of the Cognitive Science Society (pp. 60-65). Mahwah, NJ: Erlbaum.

Biederman, I. (1987). Recognition-by-components: A theory of human image understanding. Psychological Review, 94, 115-147.
Biederman, I. (1995). Visual object recognition. In S. M. Kosslyn \& D. N. Osherson (Eds.), Visual cognition: An invitation to cognitive science (2nd ed., pp. 121-165). Cambridge, MA: MIT Press.

Biederman, I., \& Cooper, E. E. (1991). Priming contour-deleted images: Evidence for intermediate representations in visual object recognition. Cognitive Psychology, 23, 393-419.

Biederman, I., Subramaniam, S., Bar, M., Kalocsai, P., \& Fiser, J. (1999). Subordinate-level object classification reexamined. Psychological Research, 62, 131-153.

Biederman, I., Subramaniam, S., Kalocsai, P., \& Bar, M. (1999). Viewpoint-invariant information in subordinate-level object classification. In D. Gopher \& A. Koriat (Eds.), Attention and performance XVII: Cognitive regulation of performance. Interaction of theory and application (pp. 91-111). Cambridge, MA: MIT Press.

Boucart, M., \& Humphreys, G. W. (1992). Global shape cannot be attended without object identification. Journal of Experimental Psychology: Human Perception \& Performance, 18, 785-806.

Boucart, M., Humphreys, G. W., \& Lorenceau, J. (1995). Automatic access to object identity: Attention to global information, not to particular physical dimensions, is important. Journal of Experimental Psychology: Human Perception \& Performance, 21, 584-601.

Bülthoff, H. H., \& Edelman, S. (1992). Psychophysical support for a two-dimensional view theory of object recognition. Proceedings of the National Academy of Sciences, 89, 60-64.

Collin, C. A., \& McMullen, P. A. (2005). Subordinate-level categorization relies on high spatial frequencies to a greater degree than basiclevel categorization. Perception \& Psychophysics, 67, 354-364.

Duncan, J., \& Humphreys, G. W. (1989). Visual search and stimulus similarity. Psychological Review, 3, 433-458.

Edelman, S., \& Duvdevani-Bar, S. (1997). A model of visual recognition and categorization. Philosophical Transactions of the Royal Society of London: Series B, 352, 1191-1202.

EDELMAN, S., \& INTRATOR, N. (2003). Towards structural systematicity in distributed, statically bound visual representations. Cognitive Science, 27, 73-109.

Filoteo, J. V., Friedrich, F. J., \& Stricker, J. L. (2001). Shifting attention to different levels within global-local stimuli: A study of normal participants and a patient with temporal-parietal lobe damage. Cognitive Neuropsychology, 18, 227-261.

Fiser, J., Subramaniam, S., \& Biederman, I. (2001). Size tuning in the absence of spatial frequency tuning in object recognition. Vision Research, 41, 1931-1950.

Han, S., \& Humphreys, G. W. (2002). Segmentation and selection contribute to local processing in hierarchical analysis. Quarterly Journal of Experimental Psychology, 55A, 5-21.

Han, S., Humphreys, G. W., \& Chen, L. (1999a). Parallel and competitive processes in hierarchical analysis: Perceptual grouping and encoding of closure. Journal of Experimental Psychology: Human Perception \& Performance, 25, 1411-1432.

Han, S., Humphreys, G. W., \& Chen, L. (1999b). Uniform connectedness and classical Gestalt principles of perceptual grouping. Perception \& Psychophysics, 61, 661-674.

Hoffman, D. D., \& Richards, W. A. (1984). Parts of recognition. Cognition, 18, 65-96.

Hoffman, D. D., \& Singh, M. (1997). Salience of visual parts. Cognition, 63, 29-78.

HüBNER, R. (2000). Attention shifting between global and local target levels: The persistence of level-repetition effects. Visual Cognition, 7, 465-484.

Hummel, J. E. (2000). Where view-based theories break down: The role of structure in human shape perception. In E. Dietrich \& A. B. Markman (Eds.), Cognitive dynamics: Conceptual and representational change in humans and machines (pp. 157-185). Mahwah, NJ: Erlbaum.

Humphreys, G. W., \& Boucart, M. (1997). Selection by color and form in vision. Journal of Experimental Psychology: Human Perception \& Performance, 23, 136-153.

Jolicceur, P., Gluck, M. A., \& Kosslyn, S. M. (1984). Pictures and names: Making the connection. Cognitive Psychology, 16, 243-275.

Kim, N., Ivry, R. B., \& Robertson, L. C. (1999). Sequential priming in hierarchically organized figures: Effects of target level and target 
resolution. Journal of Experimental Psychology: Human Perception \& Performance, 25, 715-729.

KIMCHI, R. (1998). Uniform connectedness and grouping in the perceptual organization of hierarchical patterns. Journal of Experimental Psychology: Human Perception \& Performance, 24, 1105-1118.

KIMCHI, R. (2000). Perceptual organization of visual objects: A microgenetic analysis. Vision Research, 40, 1333-1347.

LAMB, M. R., \& RoberTson, L. C. (1988). The processing of hierarchical stimuli: Effects of retinal locus, locational uncertainty, and stimulus identity. Perception \& Psychophysics, 44, 172-181.

LAMB, M. R., \& Yund, E. W. (1996). Spatial frequency and attention: Effects of level-, target-, and location-repetition on the processing of global and local forms. Perception \& Psychophysics, 58, 363-373.

LAMB, M. R., \& YUND, E. W. (2000). The role of spatial frequency in cued shifts of attention between global and local forms. Perception \& Psychophysics, 62, 753-761.

Lloyd-Jones, T. J., \& Humphreys, G. W. (1997a). Categorizing chairs and naming pears: Category differences in object processing as a function of task and priming. Memory \& Cognition, 25, 606-624.

Lloyd-Jones, T. J., \& Humphreys, G. W. (1997b). Perceptual differentiation as a source of category effects in object processing: Evidence from naming and object decisions. Memory \& Cognition, 25, 18-35.

LofTUS, G. R., \& MAsson, M. E. J. (1994). Using confidence intervals in within-subject designs. Psychonomic Bulletin \& Review, 1, 476-490.

MacInNes, J. (2000). Experiment presentation software [Computer program]. Halifax: Dalhousie University. Available at www.cs.dal .ca/ macinnwj.

Marr, D. (1982). Vision. San Francisco: Freeman.

Morrison, D. J., \& Schyns, P. G. (2001). Usage of spatial scales for the categorization of faces, objects, and scenes. Psychonomic Bulletin \& Review, 8, 454-469.

Murphy, G. L., \& Brownell, H. H. (1985). Category differentiation in object recognition: Typicality constraints on the basic category advantage. Journal of Experimental Psychology: Learning, Memory, \& Cognition, 11, 70-84.

Murphy, G. L., \& Lassaline, M. E. (1997). Hierarchical structure in concepts and the basic level of categorization. In K. Lamberts \& D. Shanks (Eds.), Knowledge, concepts, and categories (pp. 93-131). Cambridge, MA: MIT Press.

MurRay, J. E., \& Jones, C. (2002). Attention to local form information can prevent access to semantic information. Quarterly Journal of Experimental Psychology, 55A, 609-625.

NAVON, D. (1977). Forest before trees: The precedence of global features in visual perception. Cognitive Psychology, 9, 353-383.

Navon, D. (2003). What does a compound letter tell the psychologist's mind? Acta Psychologica, 114, 273-309.

Oliva, A., \& Schyns, P. G. (1997). Coarse blobs or fine edges? Evidence that information diagnosticity changes the perception of complex visual stimuli. Cognitive Psychology, 34, 72-107.

ROBERTSON, L. C. (1996). Attentional persistence for features of hierarchical patterns. Journal of Experimental Psychology: General, 125, 227-249.
Robertson, L. C., Egly, R., Lamb, M. R., \& Kerth, L. (1993). Spatial attention and cuing to global and local levels of hierarchical structure. Journal of Experimental Psychology: Human Perception \& Performance, 19, 471-487.

Robertson, L. C., \& IVRY, R. [B.] (2000). Hemispheric asymmetries: Attention to visual and auditory primitives. Current Directions in Psychological Science, 9, 59-63.

Rosch, E., Mervis, C. B., Gray, W. D., Johnson, D. M., \& BoyesBraem, P. (1976). Basic objects in natural categories. Cognitive Psychology, 8, 382-439.

SchYNS, P. G. (1998). Diagnostic recognition: Task constraints, object information and their interactions. Cognition, 67, 147-179.

Schyns, P. G., \& Oliva, A. (1999). Dr. Angry and Mr. Smile: When categorization flexibly modifies the perception of faces in rapid visual presentations. Cognition, 69, 243-265.

SnOdgrass, J. G., \& McCullough, B. (1986). The role of visual similarity in picture categorization. Journal of Experimental Psychology: Learning, Memory, \& Cognition, 12, 147-154.

Snodgrass, J. G., \& VANDERWART, M. (1980). A standardized set of 260 pictures: Norms for name agreement, image agreement, familiarity, and visual complexity. Journal of Experimental Psychology: Human Learning \& Memory, 6, 174-215.

TARR, M. J., \& BÜLTHOFF, H. H. (1995). Is human object recognition better described by geon structural descriptions or by multiple views? Comment on Biederman and Gerhardstein (1993). Journal of Experimental Psychology: Human Perception \& Performance, 21, 14941505

Tarr, M. J., \& Kriegman, D. J. (2001). What defines a view? Vision Research, 41, 1981-2004.

TARR, M. J., \& Pinker, S. (1989). Mental rotation and orientationdependence in shape recognition. Cognitive Psychology, 21, 233-282.

TARR, M. J., \& PINKER, S. (1990). When does human object recognition use a viewer-centered reference frame? Psychological Science, 1, 253-256.

Tversky, B., \& Hemenway, K. (1984). Objects, parts and categories. Journal of Experimental Psychology: General, 113, 169-193.

VAN Selst, M., \& Jolicceur, P. (1994). A solution to the effect of sample size on outlier elimination. Quarterly Journal of Experimental Psychology, 47A, 631-650.

VeCERA, S. P. (1998). Visual object representation: An introduction. Psychobiology, 26, 281-308.

WARD, L. (1982). Determinants to attention to local and global features of visual forms. Journal of Experimental Psychology: Human Perception \& Performance, $\mathbf{8}, 562-581$.

\section{NOTE}

1. A separate analysis was performed on object discriminations made in the context of visually similar distractors and those made in the context of visually dissimilar distractors. There were no significant priming effects on object discriminations made in the context of visually dissimilar distractors. 
APPENDIX A

List of Target and Distractor Objects Used in the Conditions in Experiments 1 and 2

Table A1

Test Conditions in Experiments 1 and 2

\begin{tabular}{|c|c|c|c|}
\hline $\begin{array}{l}\text { Subordinate } \\
\text { Similar }\end{array}$ & $\begin{array}{c}\text { Basic } \\
\text { Similar }\end{array}$ & $\begin{array}{c}\text { Subordinate } \\
\text { Dissimilar }\end{array}$ & $\begin{array}{c}\text { Basic } \\
\text { Dissimilar }\end{array}$ \\
\hline \multicolumn{4}{|c|}{ Targets } \\
\hline 16 finches & $\begin{array}{l}16 \text { dogs: } \\
\text { afghan } \\
\text { airedale } \\
\text { Bedlington terrier } \\
\text { bloodhound } \\
\text { Boston terrier } \\
\text { bull mastiff } \\
\text { bull terrier } \\
\text { chihuahua } \\
\text { collie } \\
\text { doberman } \\
\text { greyhound } \\
\text { husky } \\
\text { pointer } \\
\text { pug } \\
\text { retriever } \\
\text { saluki }\end{array}$ & 16 beagles & $\begin{array}{l}16 \text { fish: } \\
\text { angelfish } \\
\text { catfish } \\
\text { dogfish } \\
\text { dolphin fish } \\
\text { fighting fish } \\
\text { halibut } \\
\text { hammerhead } \\
\text { jack } \\
\text { lookdown } \\
\text { mackerel } \\
\text { ray } \\
\text { sailfish } \\
\text { salmon } \\
\text { sturgeon } \\
\text { tarpon } \\
\text { tuna }\end{array}$ \\
\hline \multicolumn{4}{|c|}{ Distractors } \\
\hline $\begin{array}{l}\text { bluejay } \\
\text { Canada goose } \\
\text { falcon } \\
\text { flamingo } \\
\text { hummingbird } \\
\text { kingfisher } \\
\text { mockingbird } \\
\text { parrot } \\
\text { pelican } \\
\text { pigeon } \\
\text { puffin } \\
\text { seagull } \\
\text { swallow } \\
\text { swan } \\
\text { titmouse } \\
\text { woodpecker }\end{array}$ & $\begin{array}{l}\text { bear } \\
\text { camel } \\
\text { cat } \\
\text { cow } \\
\text { deer } \\
\text { donkey } \\
\text { elephant } \\
\text { goat } \\
\text { gorilla } \\
\text { horse } \\
\text { lion } \\
\text { monkey } \\
\text { mouse } \\
\text { pig } \\
\text { rabbit } \\
\text { sheep }\end{array}$ & $\begin{array}{l}\text { ashtray } \\
\text { barn } \\
\text { bike } \\
\text { blouse } \\
\text { bottle } \\
\text { desk } \\
\text { gun } \\
\text { hat } \\
\text { helicopter } \\
\text { helmet } \\
\text { iron } \\
\text { oven } \\
\text { piano } \\
\text { roller skates } \\
\text { telephone } \\
\text { truck }\end{array}$ & $\begin{array}{l}\text { basket } \\
\text { bed } \\
\text { boot } \\
\text { cannon } \\
\text { car } \\
\text { church } \\
\text { coat } \\
\text { dress } \\
\text { dresser } \\
\text { guitar } \\
\text { kettle } \\
\text { kite } \\
\text { motorcycle } \\
\text { plane } \\
\text { pot } \\
\text { water can }\end{array}$ \\
\hline
\end{tabular}

Table A2

Practice Conditions in Experiments 1 and 2

\begin{tabular}{|c|c|c|c|}
\hline $\begin{array}{l}\text { Subordinate } \\
\text { Similar }\end{array}$ & $\begin{array}{l}\text { Basic } \\
\text { Similar }\end{array}$ & $\begin{array}{l}\text { Subordinate } \\
\text { Dissimilar }\end{array}$ & $\begin{array}{c}\text { Basic } \\
\text { Dissimilar } \\
\end{array}$ \\
\hline \multicolumn{4}{|c|}{ Targets } \\
\hline 6 finches & $\begin{array}{l}6 \text { dogs: } \\
\text { bulldog } \\
\text { cocker } \\
\text { German shepherd } \\
\text { Pekinese } \\
\text { sheepdog } \\
\text { basset hound }\end{array}$ & 6 beagles & $\begin{array}{l}6 \text { fish: } \\
\text { flounder } \\
\text { johndory } \\
\text { paddlefish } \\
\text { shark } \\
\text { walleye } \\
\text { bluegill }\end{array}$ \\
\hline \multicolumn{4}{|c|}{ Distractors } \\
\hline $\begin{array}{l}\text { cockatoo } \\
\text { duck } \\
\text { egret } \\
\text { meadow lark } \\
\text { stork } \\
\text { thrasher }\end{array}$ & $\begin{array}{l}\text { kangaroo } \\
\text { raccoon } \\
\text { rhinoceros } \\
\text { squirrel } \\
\text { zebra } \\
\text { beaver }\end{array}$ & $\begin{array}{l}\text { baby carriage } \\
\text { chair } \\
\text { hammer } \\
\text { lamp } \\
\text { record player } \\
\text { jet fighter }\end{array}$ & $\begin{array}{l}\text { couch } \\
\text { house } \\
\text { key } \\
\text { sailboat } \\
\text { train } \\
\text { coffee pot }\end{array}$ \\
\hline
\end{tabular}




\section{APPENDIX B}

\section{Analysis of the Attention-Priming Task With Hierarchical Stimuli in Experiment 1}

\section{Reaction Times}

The speed of detecting targets at global and local levels depended on the size of the hierarchical figures, as was evident from an interaction between size (large, medium, or small) and attention level (global or local) $[F(2,92)=3.11, p=.05]$. For large hierarchical figures, local-level targets $(M=611 \mathrm{msec})$ were identified more quickly than global-level targets $(M=617 \mathrm{msec})$. For medium hierarchical figures, there was little difference between the identification times for global and local targets. For the small hierarchical figures, global-level targets $(M=616 \mathrm{msec})$ were identified more quickly than local-level targets $(M=638 \mathrm{msec})[t(1,187)=2.48$, $p=.02]$. There were no other significant interactions or main effects.

\section{Percentages Correct}

There were more correct responses made to global and local levels of the hierarchical figures when they were preceded by object discriminations made in the context of visually dissimilar distractors $(95 \%)$ than when preceded by object discriminations made in the context of visually dissimilar distractors $(91 \%)[F(1,46)=38.92$, $p<.0001]$. There was evidence that the degree of visual similarity between targets and distractors influenced performance on global and local decisions, as was shown by an interaction between distractor type and attention level $[F(1,46)=6.86, p=.01]$. Performance on the global/local divided attention task was also influenced by the size of the hierarchical figures and the category level of the target objects $[F(2,92)=6.657, p=.002]$. In general, the responses to global- and local-level targets appeared to be influenced by the difficulty of the task.

\section{APPENDIX C Analysis of the Attention-Priming Task With Separate Digits in Experiment 2}

\section{Reaction Times}

The speed of responding to the digits depended on the size group to which the digits belonged $[F(2,94)=$ $4.78, p=.01]$. For the small digit group, global elements $(M=560 \mathrm{msec})$ were identified more quickly than local elements $(M=578 \mathrm{msec})[t(1,191)=3.87, p=.0003]$. For the large and medium digit groups, there were no reliable differences between the identification times for global and local elements $(t<1)$. There were no other significant interactions.

\section{Percentages Correct}

More correct responses were made when digit responses were preceded by object discriminations in the context of visually dissimilar distractors $(95 \%)$ than in the context of visually similar distractors $(91 \%)[F(1,47)=$ $26.47, p<.00001]$. Performance on the attention-priming task was sensitive to the category level of the preceding target object and the degree of visual similarity between the targets and the distractors $[F(1,47)=5.03, p=$ $.03]$. The percentage of correct responses also depended on the category level of the preceding target object $[F(1,47)=4.36, p=.04]$. There were more correct responses to local element decisions when they were preceded by basic object discriminations $(95 \%)$ than when preceded by subordinate object discriminations $(93 \%)$ $[t(1,287)=2.71, p=.01]$. Global element decisions were unaffected by preceding target decision $(t<1)$. According to the attentional window hypothesis, there should be more correct responses after subordinate object discriminations, since the size of the attentional window should be closer to the local than to the global digit. It could be that the pattern of correct responses has more to do with the level of difficulty experienced in preceding object discrimination trials. The fact that the degree of visual similarity between the targets and the distractors in the object discrimination task had a consistent effect on the number of correct responses to digits supports this argument.

(Manuscript received August 17, 2004;

revision accepted for publication August 26, 2005.) 